\title{
A Systematic Review and Meta-Analysis of Transcranial Direct Current Stimulation to Remediate Age-Related Cognitive Decline in Healthy Older Adults
}

This article was published in the following Dove Press journal:

Neuropsychiatric Disease and Treatment

\author{
Aprinda Indahlastari $\mathbb{D}^{1,2}$ \\ Cheshire Hardcastle ${ }^{1,2}$ \\ Alejandro Albizu (iD) ${ }^{1,3}$ \\ Stacey Alvarez-Alvarado ${ }^{1,2}$ \\ Emanuel M Boutzoukas ${ }^{1,2}$ \\ Nicole D Evangelista ${ }^{1,2}$ \\ Hanna K Hausman (iD) ${ }^{1,2}$ \\ Jessica Kraft ${ }^{1,3}$ \\ Kailey Langer (iD) ${ }^{1,2}$ \\ Adam J Woods ${ }^{1-3}$ \\ 'Center for Cognitive Aging and \\ Memory, McKnight Brain Institute, \\ University of Florida, Gainesville, FL, \\ USA; ${ }^{2}$ Department of Clinical and Health \\ Psychology, University of Florida, \\ Gainesville, FL, USA; ${ }^{3}$ Department of \\ Neuroscience, University of Florida, \\ Gainesville, FL, USA
}

Correspondence: Aprinda Indahlastari Department of Clinical and Health Psychology, University of Florida,

Gainesville, FL, USA

Tel +l 352-294-5842

Email aprinda.indahlas@phhp.ufl.edu
Background: Transcranial direct current stimulation (tDCS) has been proposed as a possible method for remediating age-associated cognitive decline in the older adult population. While tDCS has shown potential for improving cognitive functions in healthy older adults, stimulation outcomes on various cognitive domains have been mixed.

Methods: A systematic search was performed in four databases: PubMed, EMBASE, Web of Science, and PsychInfo. Search results were then screened for eligibility based on inclusion/exclusion criteria to only include studies where tDCS was applied to improve cognition in healthy older adults 65 years and above. Eligible studies were reviewed and demographic characteristics, tDCS dose parameters, study procedures, and cognitive outcomes were extracted. Reported effect sizes for active compared to sham group in representative cognitive domain were converted to Hedges' g.

Main Results: A total of thirteen studies involving healthy older adults $(n=532$, mean age $=71.2+5.3$ years) were included in the meta-analysis. The majority of included studies (94\%) targeted the prefrontal cortex with stimulation intensity 1-2 mA using various electrode placements with anodes near the frontal region. Across all studies, we found Hedges' $g$ values ranged from -0.31 to 1.85 as reported group effect sizes of active stimulation compared to sham.

Conclusion: While observed outcomes varied, overall findings indicated promising effects of tDCS to remediate cognitive aging and thus deserves further exploration. Future characterization of inter-individual variability in tDCS dose response and applications in larger cohorts are warranted to further validate benefits of tDCS for cognition in healthy older adults.

Keywords: tES, tDCS, aging, cognitive decline

\section{Introduction}

The population of older adults over the age of 65 is one of the fastest growing demographics in the United States and is expected to double by the year $2050 .^{1}$ As this subset of the population grows, research has focused on improving quality of

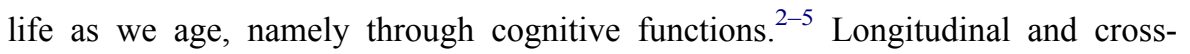
sectional studies tracking cognitive functions have identified a pattern of decline in cognitive domains of attention, processing speed, executive functioning, and episodic and semantic memory as a function of age. ${ }^{6,7}$ On the other hand, cognitive 
abilities such as vocabulary are resilient to brain aging, and may even improve with age. ${ }^{8}$ This age-associated pattern of change is referred to as cognitive aging. ${ }^{9-11}$ As cognitive skills decline, the rate of functional dependence, mortality, and acute illness requiring hospitalization increases. ${ }^{12,13}$ In order to offset the trajectory of cognitive decline in the aging population, researchers have explored methods of intervention, such as non-invasive brain stimulation, to maintain or improve cognitive functions sensitive to aging.

Transcranial direct current stimulation (tDCS) is a promising non-invasive brain stimulation technique involving the delivery of a weak electrical current (1-2 $\mathrm{mA}$ ) to the scalp via surface electrodes, modulating neuronal membrane potentials. ${ }^{14-19}$ tDCS has been used as a cognitive intervention technique by strategically placing electrodes over targeted brain areas vulnerable to cognitive aging, such as the prefrontal cortex, to strengthen synaptic signaling, thereby improving executive functioning, working memory, and processing speed performance. ${ }^{20-28}$ However, the exact mechanism by which tDCS enhances cognitive functions is not yet well understood, as multiple brain areas are recruited to execute and perform cognitive tasks. ${ }^{29}$ One explanation is that tDCS stimulation of one brain structure (eg, the dorsolateral prefrontal cortex) may induce increased connectivity of brain networks (eg, Default Mode Network) associated with cognitive function. ${ }^{30-32}$ Improvements in cognitive functioning via tDCS may also transfer to improved functional abilities crucial for activities of daily living. ${ }^{33-37}$

Despite the growing body of research suggesting tDCS is an effective intervention to remediate cognitive decline, ${ }^{16,29,38-40}$ few studies have reviewed its efficacy in a healthy aging population. The literature has yielded mixed findings among the studies reviewing cognitive improvement after tDCS in young adult populations. For instance, findings from a quantitative review by Horvath et $\mathrm{al}^{41}$ did not support the efficacy of single session tDCS in healthy young adult populations. Some have suggested this reflects a ceiling effect of potential cognitive gains in healthy adults. ${ }^{39,40}$ However, another systematic review performed by Dedoncker et $\mathrm{al}^{38}$ found single session tDCS to have a modest effect in improving speed of response in healthy young adults, specifically in those studies that applied larger current density (current intensity per-unit-area). In a systematic review involving an older adult population, a meta-analysis by Summers et $\mathrm{al}^{29}$ found enhanced cognitive performance across multiple cognitive domains and stimulation parameters (eg, offline versus online stimulation). This suggests that tDCS efficacy may be different in an older adult population compared to a young adult population. However, the magnitude of cognitive benefits was observed to be different between online (stimulation during task performance) and offline (stimulation before task performance), suggesting that the cognitive benefit of tDCS may interact with stimulation parameters and timing of stimulation delivery. Importantly, Summers et $\mathrm{al}^{29}$ investigated studies that included adults below the age of 65 . Thus, whether these conclusions apply to an older adult population has yet to be determined. Other systematic reviews have surveyed potential effects of tDCS in cognitively impaired aging populations (eg, Alzheimer's disease) and demonstrated slight improvements in cognitive functioning in these populations after tDCS. ${ }^{42,43}$ Both types of systematic review studies covering tDCS as an intervention to improve cognition in both cognitively healthy and impaired older adults suggested that tDCS may be an effective method of intervening in age-related cognitive decline. However, drawing a cohesive conclusion of tDCS effects on cognitive aging remains a challenge due to the lack of consistency in outcomes reported from tDCS applications in mixed populations of older adults (healthy or otherwise). Further, the current state of research investigating tDCS application to remediate cognitive function employs a wide array of tDCS parameters and assesses various behavioral outcomes despite targeting the same cognitive domains that need to be addressed.

Previous literature has shown that there is reliable agerelated decline in areas of attention, processing speed, executive functioning, and episodic and semantic memory. ${ }^{6}$ Structural equation modeling suggests that while speed/attention and episodic memory decline at a greater magnitude in the cognitive trajectory, ${ }^{44} 46$ these individual cognitive domains form a dynamic relationship with one another. ${ }^{47}$ For example, previous longitudinal studies have identified perceptual processing as a driving factor in decline of memory. ${ }^{48}$ The cognitive domains selected in this review therefore include tasks targeting attention (sustained attention, visual attention), executive functioning (error awareness, working memory), and episodic memory. Brain areas that play a role in these cognitive functions are also target sites for tDCS (eg, dorsolateral prefrontal cortex), and thus tDCS intervention could be an appropriate method to improve performance in these cognitive domains that decline with age, as 
suggested in previous literature. Further, in addition to the fast growing population of older adults aged 65 and over worldwide, ${ }^{49,50}$ the Center for Disease Control has indicated that after age 65 there is a sharp increase in subjective cognitive complaints and cognitive decline that has been deemed a public health issues. ${ }^{51}$ Additionally, the World Population Ageing 2019 Highlights by the United Nations refer to the older adult population as 65 years and older. ${ }^{52}$ Further, while there are a number of interventions targeting adults in the age range of 60 and older, prior cross-sectional research suggests the largest change in agerelated cognitive decline will likely become apparent in the age range of $60-70$, due to accelerated decline after the age of $60 .{ }^{47}$ Given this information, the present metaanalysis study focused on a population that is representative of both older adults and adults that have started experiencing cognitive decline. Considering the growing proportion of older adults in the United States and worldwide, it is important to understand the benefits of tDCS in this population as a method to alter the trajectory of cognitive decline within the normal aging process. Moreover, it is important to consider methodical implications in the efficacy of this intervention. Therefore, this systematic review aimed to 1) assess study protocols and efficacy of tDCS to remediate cognitive functions in healthy older adults over the age of 65 , and 2) comment on potential methodological factors and publication bias in the current field that may contribute to findings.

\section{Methods}

\section{Literature Search}

Literature search was conducted on June 30, 2020 in the following databases: PubMed, EMBASE, Web of Science, and PsychInfo. The following keywords and boolean search terms were used to search in title and abstract only, with formatting specifically tailored to each database: (((“tDCS"[Title/Abstract]) OR ("transcranial direct current stimulation"[Title/Abstract]) OR ("direct stimulation"[Title/ Abstract]) OR ("transcranial electrical stimulation"[Title/ Abstract])) AND ((“cognition"[Title/Abstract]) OR ("cognitive"[Title/Abstract]) OR ("memory"[Title/Abstract]) OR ("speed of processing"[Title/Abstract]) OR ("brain function"[Title/Abstract]) OR (“decision making”[Title/ Abstract]) OR (“'attention"[Title/Abstract])) AND ((“aging”[Title/Abstract]) OR (“ageing"[Title/Abstract]) OR (“older”[Title/Abstract]) OR (“elderly”[Title/Abstract]) OR (“geriatric"[Title/Abstract]) OR (“old age"[Title/Abstract]))).
There were no date restrictions used in the search, and thus all published studies up to the search date were considered in the screening process. The PRISMA checklist (Appendix A) was used to conduct the present systematic review.

\section{Study Eligibility}

Lists of articles obtained from each literature search database were exported as research information system (RIS) files and imported to Covidence (https://www.covidence. org/) for abstract and full text screening. The screening process was performed by two independent reviewers per article. In Covidence, duplicate entries were identified and removed, then abstract screening was performed to select studies according to the inclusion/exclusion criteria (Table 1). Relevant articles that passed the abstract screening were entered for a full text screening. In this stage, eligible papers were thoroughly read, and re-classified based on inclusion/exclusion criteria. The included articles were written in English and utilized a randomized or pseudorandomized controlled trial (RCT) design with pre- and post-assessment. Observational studies, review articles, published abstracts, and case-studies were excluded. Study populations were restricted to older adults (age $\geq 65$ ) without neurological or psychiatric diagnoses or impairments. Articles including samples with mild cognitive impairment or dementia were excluded. Participants needed to receive tDCS with sham-controlled comparisons. Stimulation modality had to be exclusively tDCS, regardless of montage. To minimize confounding variables, intervention protocols could not include pharmacological or combined brain stimulation techniques. Lastly, studies must have clearly

Table I Inclusion and Exclusion Criteria Used in the Article Screening Process

\begin{tabular}{|c|c|}
\hline Inclusion & Exclusion \\
\hline - Age $\geq 65$ & \multirow{2}{*}{$\begin{array}{l}\text { - Diagnosis of neurological or } \\
\text { psychiatric diagnosis or impair- } \\
\text { ments, or major neurocognitive } \\
\text { disorder }\end{array}$} \\
\hline $\begin{array}{l}\text { - Randomized or pseudo- } \\
\text { randomized controlled trial } \\
\text { (active and sham) }\end{array}$ & \\
\hline $\begin{array}{l}\text { - tDCS as the stimulation tech- } \\
\text { nique for intervention }\end{array}$ & \multirow{2}{*}{$\begin{array}{l}\text { - Using tDCS in combination } \\
\text { with other stimulation } \\
\text { techniques }\end{array}$} \\
\hline $\begin{array}{l}\text { - Cognition as primary mea- } \\
\text { sured outcome }\end{array}$ & \\
\hline $\begin{array}{l}\text { - Cognitively intact or cogni- } \\
\text { tively normal participants }\end{array}$ & \multirow[t]{2}{*}{$\begin{array}{l}\text { - Observational studies, review } \\
\text { articles, published abstracts }\end{array}$} \\
\hline - Written in English & \\
\hline
\end{tabular}




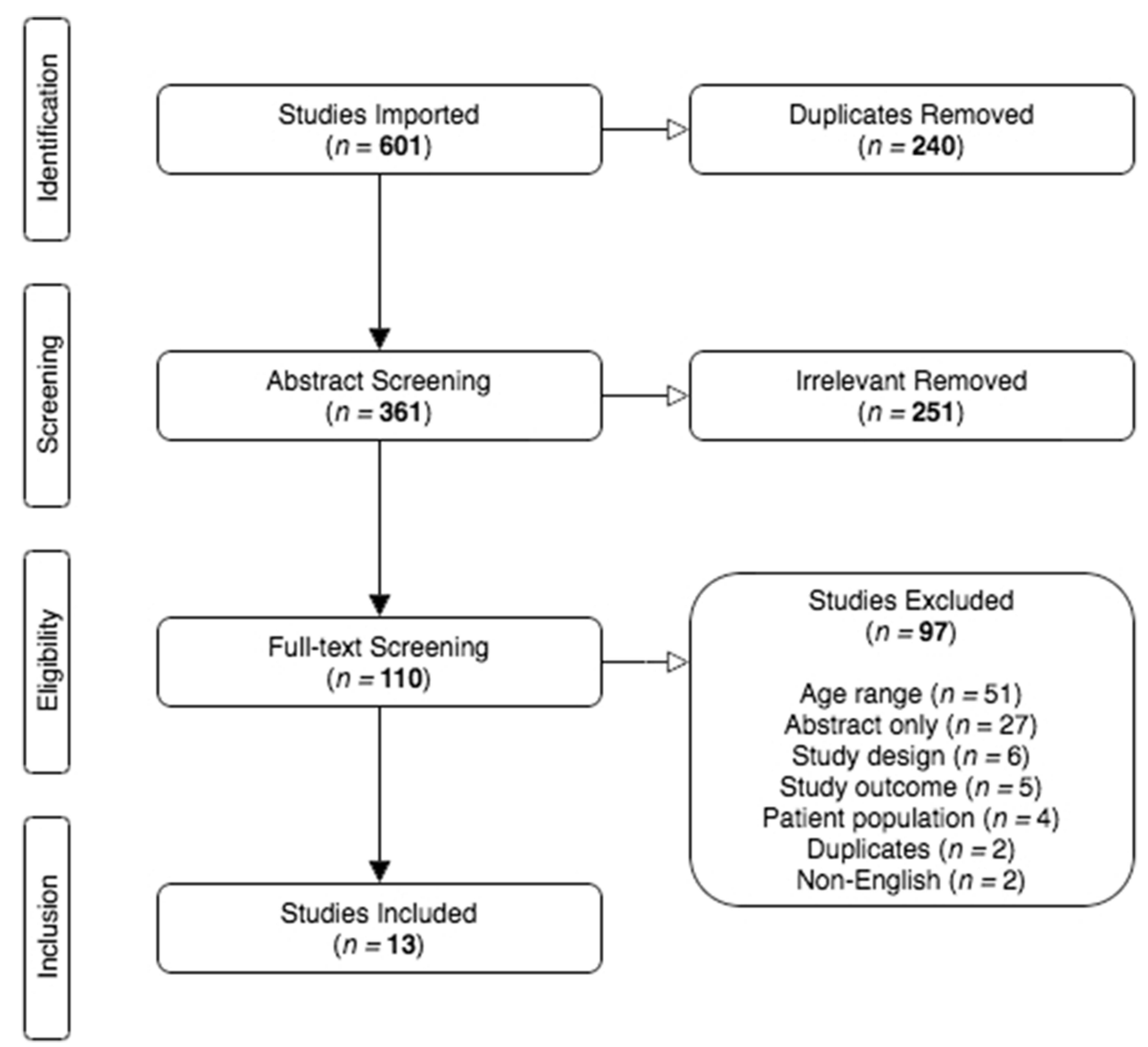

Figure I PRISMA diagram illustrates search results for each stage of the systematic review process.

Notes: Adapted from Moher D, Liberati A., Tetzlaff J, Altman DG. Preferred reporting items for systematic reviews and meta-analyses: The PRISMA statement.PLoS Medicine. 2009;6(7):el000097. Creative Commons license and disclaimer available from: http://creativecommons.org/licenses/by/4.0/legalcode ${ }^{120}$

established their primary outcome as cognitive performance. Any excluded, irrelevant, and removed articles were tracked in the PRISMA diagram (Figure 1). Included articles were then entered into Google Scholar and Web of Science for "snowballing" procedure. This process involved identifying other papers that cited the included articles (backward "snowballing") and searching the references within the included articles (forward "snowballing") for potential studies not retrieved in the initial search.

\section{Data Extraction}

Pairs of authors reviewed the full texts of the final sample of studies to extract the following information: demographic characteristics (age, sex, years of education, cognitive screening performance), tDCS parameters (stimulation intensity, duration, and number of sessions; target brain region; electrode size, montage, and wash-out period), study procedures (sample size, blinding procedures, cognitive domain), and results of stimulation (outcome measures, effect sizes). The inter-rater reliability of each pair is reported in Appendix B.
Any disputed studies were discussed among authors to reach a consensus. All reported effect sizes $(p<0.05)$ between active and sham group were converted to Hedges' $g$ for comparison across studies. Subsequently, one author (AI) confirmed the accuracy of the data extraction.

\section{Outcome Variables}

Cognitive domains of sustained attention, visual attention, error awareness, verbal episodic memory, and working memory were identified in the included studies. Specific outcome measures corresponding to these cognitive domains were also extracted from each study.

\section{Quality Assessment}

The Revised Cochrane Risk of Bias Tool 2 (RoB2) was utilized to assess risk of bias of all full text studies included. ${ }^{53}$ This tool was designed to determine risk of bias of randomized controlled trials using intent-to-treat analyses. For the purposes of this tool, bias is defined as a systematic deviation from intended intervention. ${ }^{54}$ The 
RoB2 tool risk-of-bias ratings are characterized by five domains developed to indicate different stages of randomized controlled trials where bias may be introduced: ${ }^{54}$

1. bias arising from the randomization process assesses the randomization process of allocation and allocation concealment.

2. Bias due to deviations from intended interventions assesses deviations from the intended protocol and if these deviations could cause bias to reported results.

3. Bias due to missing outcome data - bias due to missing data (eg, intention-to-treat analysis, imputations, bias in drop-out and attrition, imputation).

4. Bias in measurement of the outcome - errors due to bias in outcome variables that could be due to misclassification, measurement errors, or errors related to intervention assignment.

5. Bias in selection of the reported result - bias due to reported results being selected among multiple options, or incomplete reporting.

Through use of their programmed excel sheet and algorithm, "signaling" questions with answers of yes, probably yes, no, probably, no, or no information, determined risk (low risk, some concerns, or high risk) in each domain. Then, each paper was given an overall risk score based on domain ratings. Each full text document was assessed independently by two reviewers. A consensus was made on discrepant scores.

\section{Statistical Analysis}

The "meta" package in R software (v4.0.3) was used to perform all statistical analyses and generate all plots (a forest plot and two funnel plots). Hedges' $g$ was used as a measure of effect size by taking the difference in group mean $(\bar{y})$ divided by the pooled standard deviation $\left(s_{p}\right)$ as noted in the formula below:

$$
g=\frac{\bar{y}_{1}-\bar{y}_{2}}{s_{p}}
$$

The following correction for small sample was also applied where appropriate:

$$
\frac{n-3}{n-2.25} \sqrt{\frac{n-2}{n}}
$$

The Chi-squared test was used to compute heterogeneity for each outcome and the Egger's test ${ }^{55}$ was used to assess risk of publication bias. The Duval and Tweedie's trim and fill procedure $^{56,57}$ was performed as needed to correct for publication bias. To assess the potential effects of dose on treatment effects, meta-regression analyses were performed to assess the continuous, linear relationships between observed total effect sizes and tDCS dosing parameters (ie, a combination of current intensity, duration and electrode surface area) as well as timing (online/offline), laterality and age. Due to the limited sample of the current meta-analysis $(\mathrm{k}=17)$, permutation tests with 1000 iterations were performed to generate robust meta-regression estimates.

\section{Results}

\section{Study Overview}

Figure 1 shows the PRISMA diagram that summarizes article search and screening results. The search query across four databases yielded 601 studies with 240 duplicates removed, totaling 361 studies ready for screening. Abstract screening reduced the number of studies from 361 to 110 . Full text review following our inclusion and exclusion criteria eliminated 97 studies due to age requirement (51), abstract only (27), study design (6), study outcome (5), patient population (4), duplicates (2), and written in language other than English (2). There was only one eligible study harvested from the "snowballing" results. Two studies reported more than one tDCS experiment in the same paper and thus each experiment was separated in reported tables, with a total of 17 tDCS experiments enrolling 532 participants.

\section{Quality Assessment}

Risk of bias summary following the Cochrane bias tool is reported in Figure 2. The overall rating for bias risks indicated some concerns of bias in eleven and high risk in two studies. Some concerns of bias in each domain largely resulted from lack of information. For instance, there was no explicit description of whether the data analysis was performed prior to unblinding procedure that resulted in some concerns of bias in category 5 (selection of the reported results) for all included studies. Further, 59\% of studies were conducted in single-blinded manner that contributed to potential bias in category 2 (deviations from intended interventions). Most studies did not have any missing outcome data, except one study (Medvedeva et al, 2019) that analyzed 22 out of 24 enrolled participants due to dropout and technical failures. One study (Cespón et al, 2017) did not report the blinding procedure and thus resulting high-risk bias in category 2 (deviations from intended interventions). 


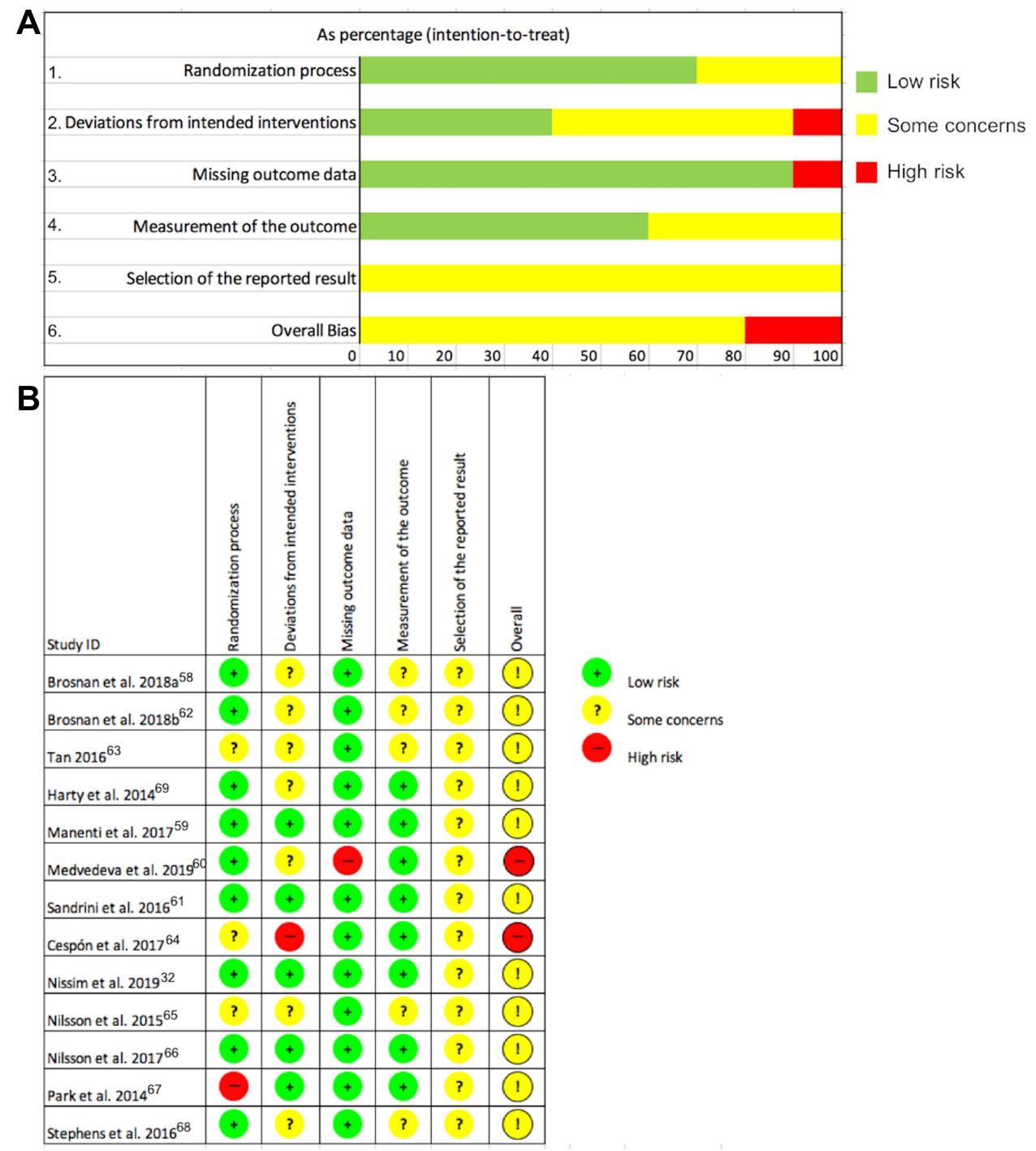

Figure 2 Summary of quality assessment using the Cochrane Risk Bias Tool. ${ }^{54}$ (A) Risk of bias graph showing overall risks across thirteen studies. (B) Risk of bias ratings for individual studies.

\section{Study Population}

Details on the population enrolled in each of the thirteen included studies are summarized in Table 2. The overall average age across the thirteen investigated studies was 71.2+5.3 years (range: $65-88$ years), which reflects the similarity in study population in terms of chronological age. The majority of studies enrolled more female participants (overall average ratio: 291 females, 204 males), with one study (Tan 2016) enrolling equal amounts of female and male participants, and one study (Brosnan et al, $2018 \mathrm{~b}$ ) omitted this information. $31 \%$ of the studies utilized the Montreal Cognitive Assessment (MoCA) (average reported score: $27.58+1.78$ ) while 54\% used the Mini Mental State Examination (MMSE) (average reported score: $28.64+1.19)$ to screen participants for cognitive status. The MMSE and MoCA are standardized screening tests to evaluate global cognitive impairments. These scores were used to determine that the study population was cognitively healthy and thus satisfied our inclusion criteria. While two studies (Cespón et al, 2017 and Nilsson et al, 2015) did not provide any details regarding a cognitive screening process used to determine participants' cognitive state, they stated that their study populations were deemed cognitively healthy, which met our eligibility criteria. The years of education across thirteen studies were averaged to $14 \pm 3.7$ years.

\section{tDCS Protocols}

All studies included sham as the control group, with $41 \%$ of the studies using a between-subject design and 59\% using 


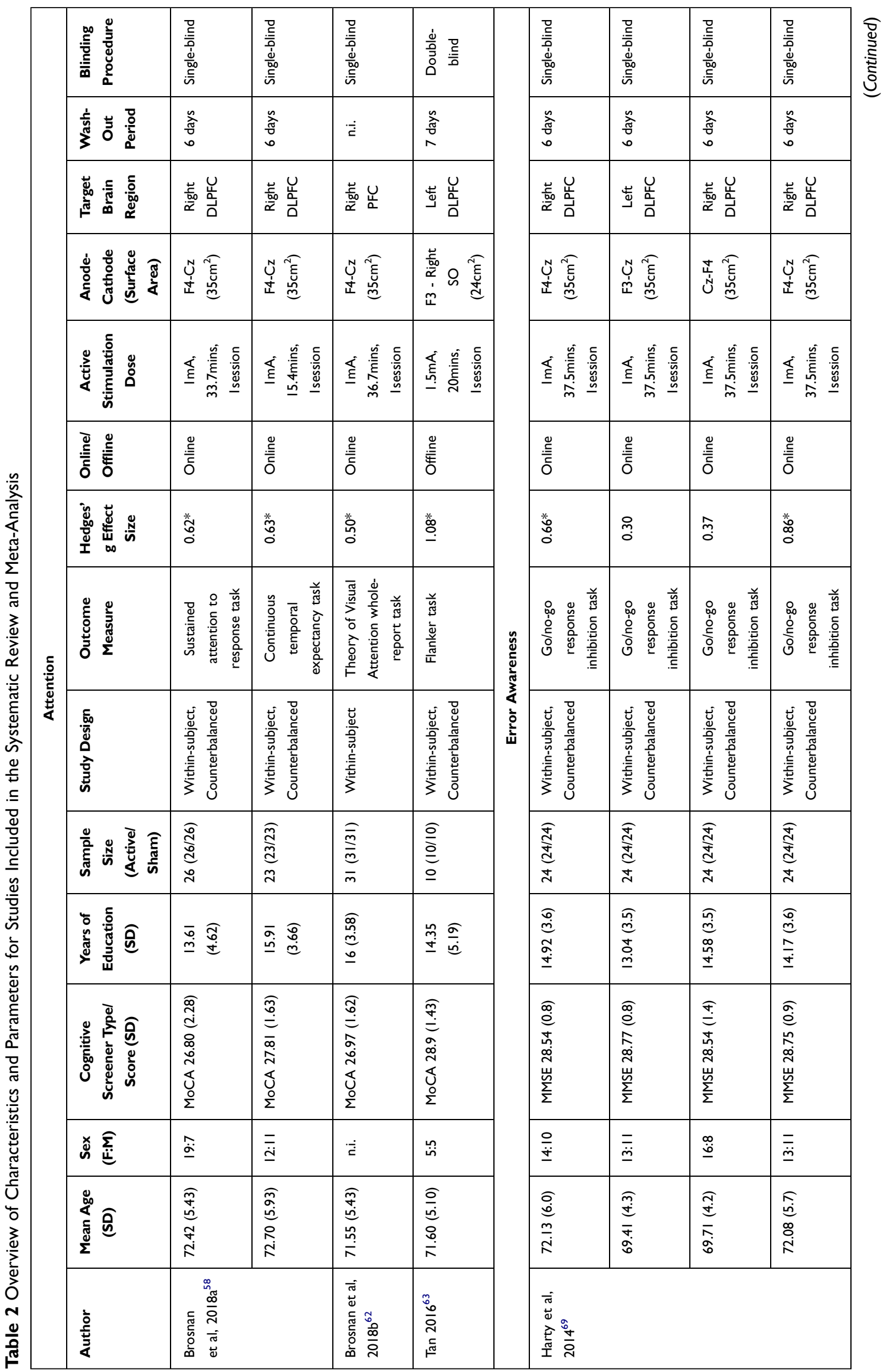




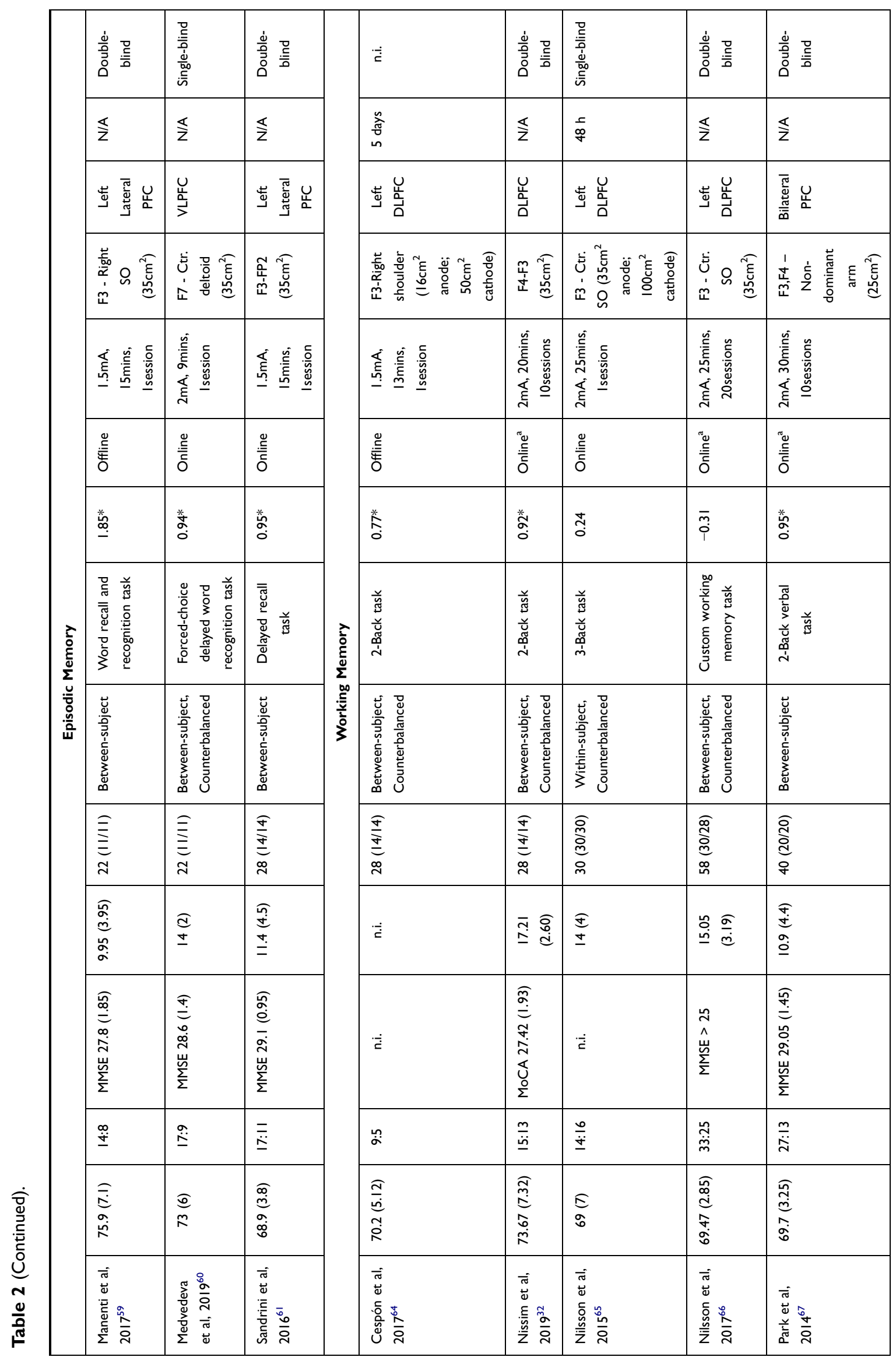




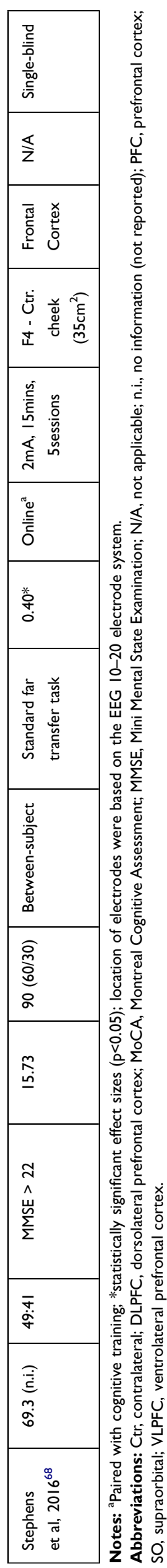

a within-subject/crossover study design. Table 2 provides a summary of tDCS protocols used across the thirteen studies. Overall, all studies were targeting the frontal cortex as the stimulated region. Specifically, $94 \%$ of the studies targeted the prefrontal cortex (PFC) with further breakdown of $64 \%$ targeting the dorsolateral PFC (DLPFC), $6 \%$ for the ventrolateral PFC (VLPFC), $12 \%$ for the left PFC, $6 \%$ for the right $\mathrm{PFC}$, and $6 \%$ bilateral/whole $\mathrm{PFC}$. One study (Stephens et al, 2016) aimed to target the entire frontal cortex. The current intensity used ranged from 1-2 mA with an average stimulation duration of 24.9 minutes. The majority of studies (13/17 studies) performed a single stimulation session. One study (Stephens et al, 2016) performed 5 sessions, two studies (Nissim et al, 2019, Park et al, 2014) performed 10 sessions, and one study (Nilsson et al, 2017) performed 20 sessions of stimulation. All studies employed the conventional tDCS set-up with a pair of large sponge or carbon rubber electrodes with an average surface area of $33 \mathrm{~cm}^{2}$ for the anode and $36 \mathrm{~cm}^{2}$ for the cathode electrodes. Washout periods for within-subject design, where each participant received both active and sham stimulation in a separate session, ranged from a minimum of $48 \mathrm{~h}$ to 6 days.

\section{Treatment Effects of tDCS}

An overview of study outcomes including cognitive domain, outcome measure, and effect size are reported in Table 2. Studies that included multiple outcome measures were simplified to include only the outcome measure most representative of tDCS effects on a domain impacted by cognitive aging. ${ }^{29}$ Details regarding each task that was used to determine each outcome measure can be found in their respective studies. ${ }^{32,58-69}$ Overall, the cognitive domains that were targeted across the thirteen studies were composed of $35 \%$ working memory, $24 \%$ attention, $23 \%$ error awareness, and $18 \%$ episodic memory. Figure 3A shows a forest plot to illustrate the weighted effect sizes in each cognitive domain. The overall weighted average of effect sizes for active over sham group across all 17 experiments was $g=0.62(\mathrm{CI}=[0.42 ; 0.84])$. The weighted average effect sizes per domain as illustrated in Figure 3A were as follows: $g=0.63(\mathrm{CI}=[0.32 ; 0.94])$ for attention, $g=0.48(\mathrm{CI}=[-0.01 ; 0.96])$ for working memory, $g=0.54(\mathrm{CI}=[0.13 ; 0.96])$ for error awareness, and $g=0.63$ $(\mathrm{CI}=[0.42 ; 0.84])$ for episodic memory. Two outliers were identified (Manenti et al, 2017 and Nilsson et al, 2017). Removing the outliers produced an overall effect size of $0.61(\mathrm{CI}=[0.469 ; 0.757], \mathrm{t}[14]=9.15, \mathrm{p}<0.0001)$. Among the included studies in this review, thirteen experiments 
Table 3 Univariate Meta-Regression Analysis

\begin{tabular}{|c|c|c|c|c|}
\hline & Omnibus ANOVA & b-Weight & $95 \% \mathrm{Cl}$ & $p$-value \\
\hline Intensity & $F[1,15]=2.1249$ & 0.0954 & {$\left[\begin{array}{lll}-0.06 & 0.21\end{array}\right]$} & 0.169 \\
\hline Density & $F[1,15]=3.4525$ & 34.99 & {$[-7.6776 .1]$} & $0.086^{\dagger}$ \\
\hline Charge & $F[1,15]=3.402$ & 1.2982 & {$\left[\begin{array}{ll}-0.22 & 2.82\end{array}\right]$} & $0.088^{\dagger}$ \\
\hline Dose & $F[1,15]=0.6258$ & 0.1516 & {$\left[\begin{array}{ll}-0.29 & 0.73\end{array}\right]$} & 0.443 \\
\hline Timing & $F[1,15]=0.3386$ & 0.0812 & {$\left[\begin{array}{ll}-0.22 & 0.38\end{array}\right]$} & 0.569 \\
\hline Laterality & $F[1,15]=0.0836$ & 0.0298 & {$\left[\begin{array}{ll}-0.19 & 0.25\end{array}\right]$} & 0.777 \\
\hline Age & $F[1,15]=10.25$ & 0.1414 & {$\left[\begin{array}{ll}0.05 & 0.24\end{array}\right]$} & $0.006 *$ \\
\hline
\end{tabular}

Notes: ${ }^{\dagger} p<0.1 ;{ }^{*} p<0.05$.

reported statistically significant effect sizes $(\mathrm{p}<0.05)$ with computed Hedges' g values ranging from -0.31 to 1.85 (mean: 0.86 \pm 0.36 ). When considering each cognitive domain, the unweighted mean and standard deviation of reported statistically significant effect sizes were 0.71 \pm 0.26 for attention, $0.76 \pm 0.14$ for error awareness, 1.25 +0.52 for episodic memory, and $0.76+0.25$ for working memory. No significant heterogeneity was observed overall $\left(\mathrm{I}^{2}=25 \%, \chi_{16}^{2}=21.42, \mathrm{p}=0.16\right)$ or within subgroup analyses (p's $>0.05$, Figure 3A). Meta-regression analyses were performed to quantify individual effects of each tDCS parameter on observed effect sizes. As shown in Table 3, none of the tDCS parameters tested was significantly related to study effect sizes ( $p$ 's $>0.05$, uncorrected) except age. Figure 3B demonstrates the relationship between effect size and age, which was the only significant predictor of observed effect size $\left(\mathrm{R}^{2}=0.3828, \mathrm{~F}\right.$ $[1,15]=10.25, \quad b$-weight $=0.14, \quad \mathrm{CI}=[0.047 ; \quad 0.236]$, $\mathrm{p}=0.006$ ).

\section{Publication Bias}

Funnel plots to illustrate analyses of publication bias are shown in Figure 4. Egger's test for asymmetry as an evaluation of publication bias revealed significant results $(\mathrm{t}[16]=2.86, \mathrm{p}=0.0119)$ that indicates evidence of publication bias in our sample. The Duval and Tweedie trim-andfill procedure showed significant results with an overall effect size of $0.49 \quad(\mathrm{CI}=[0.259 ; 0.719], \mathrm{t}[21]=4.42$, $\mathrm{p}=0.0002$ ), suggesting that after correcting for publication bias, the effect size remained significant.

\section{Discussion}

The present systematic review and meta-analysis aimed to provide a recent survey of clinical studies published until June 30, 2020 that applied tDCS to improve cognition, specifically in cognitively intact older adults of 65 years and above. Collectively, clinical studies have demonstrated
tDCS as a promising intervention strategy to combat the effects of cognitive aging for this population. We found a total of twelve eligible studies from the initial search and included an additional study from the "snowballing" process, indicating a thorough application of our search strategy to cover the literature regarding tDCS and cognitive aging. Across the thirteen eligible studies, there were 17 tDCS experiments enrolling a total of 532 participants. All studies exclusively applied tDCS as a stimulation technique to remediate cognitive decline related to normal aging. The quality assessment results indicated low to moderate concerns in five bias-risk categories in most studies, with two studies deemed as high-risk. The overall weighted average of Hedges' g across 17 experiments revealed a moderate effect size $(0.63, \mathrm{CI}=[0.42 ; 0.84])$, which is considerably larger than prior meta-analyses that included young and middle-aged adults (eg, 18 to 59 years). For instance, Dedoncker et al performed a metaanalysis of a single tDCS session targeting the DLPFC (mean age: 19.8 to 79.2 years) and found no significant effect of tDCS on accuracy (Hedges' $\mathrm{g}=0.08, \mathrm{CI}=[-0.00$; $0.17]$ ) across 61 studies including young, middle-aged, and older adults. ${ }^{38}$ In addition, we found the greatest magnitude of improvement after tDCS intervention in the studies included in this meta-analysis were in domains of episodic memory and attention (Figure 3A), which typically show the most decline in aging. ${ }^{44-46}$ Findings of the current meta-analysis are discussed further in the following subsections.

\section{Overall tDCS Effects tDCS Parameters}

While the overall statistically significant effects across studies suggest tDCS improves cognition in older adults, there is no clear distinction of which combination of current dose parameters (eg, current intensity, stimulation duration, number of sessions) yields the greatest effect in 

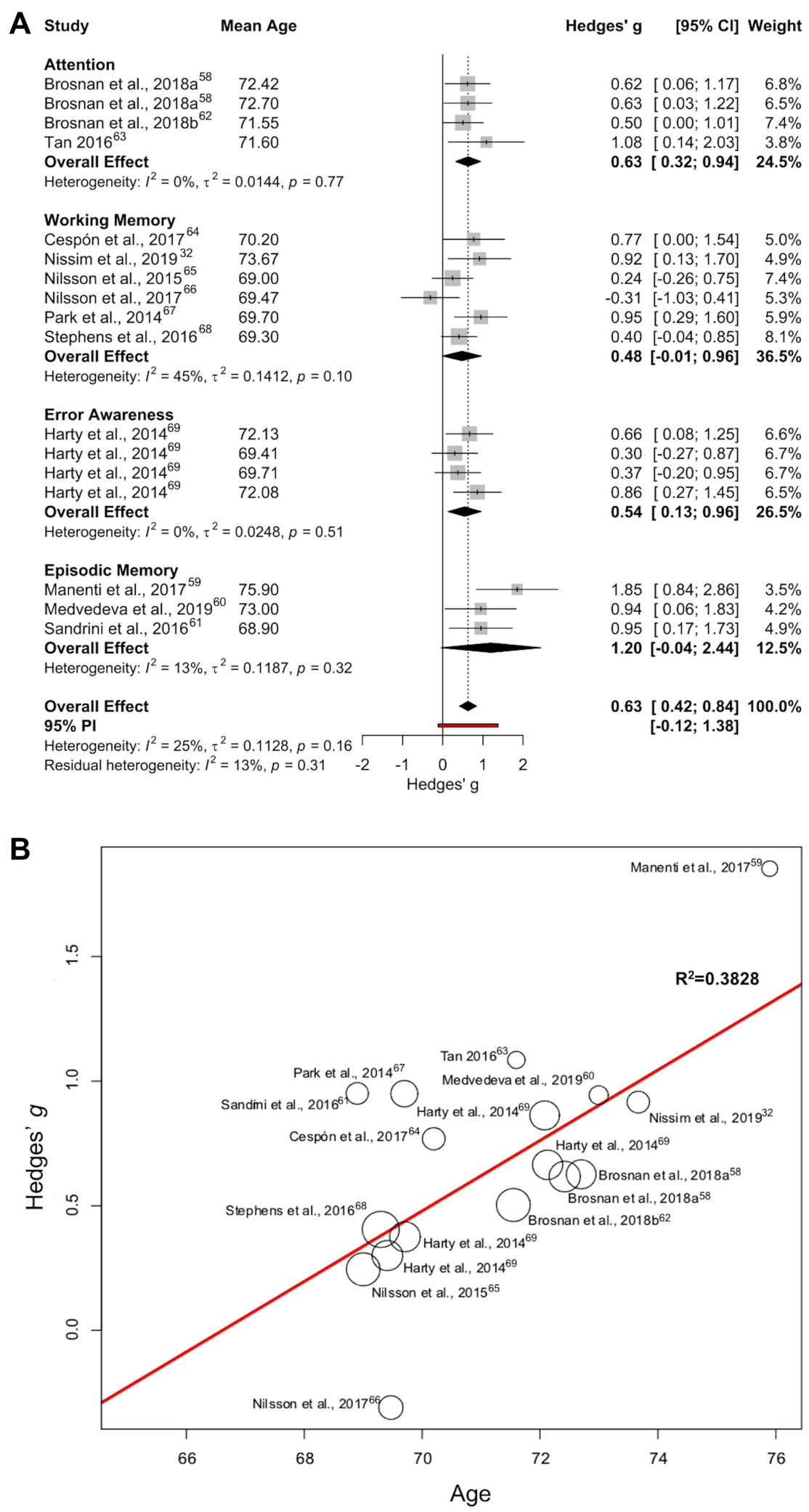

Figure 3 Forest plot and bubble plot to illustrate weighted effect sizes in all studies. (A) Forest plot categorized by each cognitive domain illustrates computed Hedges' $\mathrm{g}$ across cognitive domains. (B) Bubble plot demonstrates significant meta-regression result of age vs effect size (Hedges' g), and the location of two identified outliers (Nilsson et al, $2017^{66}$ and Manenti et al, 2017 $7^{59}$ ). Individual weights assigned to each study is indicated by the diameter of the circle. The estimated slope of this curve shows the significant effect $\left(R^{2}=0.3828, p=0.006\right)$ of mean study age on treatment effects (Hedges' g). 

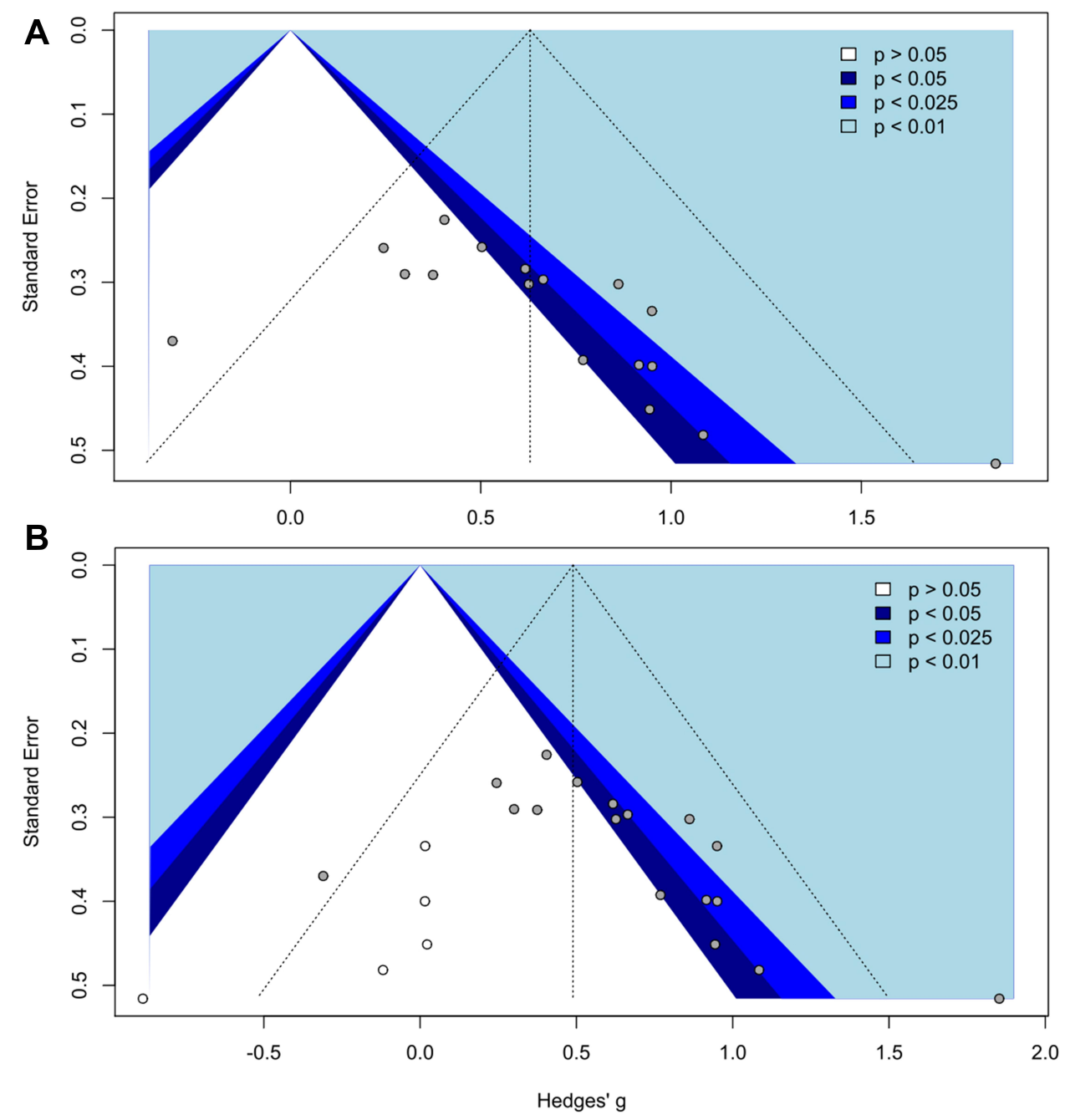

Figure 4 Funnel plots to assess publication bias. $(\mathbf{A})$ Egger's test for asymmetry $(k=17)$ indicates publication bias exists in our sample $(t[16]=2.86, p=0.0119)$. $(B)$ Duval and Tweedie trim and fill procedure $(k=22)$ to correct biases shows significant results (Hedges' $g=0.489, \mathrm{Cl}=[0.259 ; 0.719], t[21]=4.42, p=0.0002)$. Different color shades illustrate different $p$-values: $p>0.5$ (white), $p<0.05$ (dark blue), $p<0.025$ (blue), $p<0.01$ (light blue).

each cognitive domain. In the present review, tDCS application targeting the frontal cortices showed an overall effect size of $0.63 \pm 0.21$ for active over sham group across a variety of cognitive domains. The four targeted cognitive domains included in this review were attention, error awareness, episodic memory, and working memory. Cognitive performance in each domain was assessed by using a variety of outcome measures across studies (eg, 2-back task or custom working memory task to assess working memory performance). Therefore, further analyses to determine which current dose parameter is the most successful to improve a targeted cognitive domain need to be narrowed down to a single outcome measure. For example, assessing the combination between the amount of applied current and duration of stimulation within the 2-back working memory task. The 2-back task is a form of the N-back task where participants are instructed to memorize a sequence of letters and prompted whether the current presented letter matches the letter that appears two-trials back (Appendix C). ${ }^{32}$ We found the intensity of injected current was not related to observed effect sizes $\quad(b$-weight $=0.0954, \quad \mathrm{CI}=[-0.063 ; 0.214])$. Among the studies that used the 2-back task, Nissim et al versus Park et al applied 10 sessions of $2 \mathrm{~mA}$ stimulation with different stimulation duration of 20 and 30 minutes. Park et al reported a slightly larger effect size (0.95) when applying tDCS for longer (30 minutes) compared to Nissim et al (effect size: 0.92, duration: $20 \mathrm{~min}$ utes). These findings did not align with a prior study conducted by Hassanzahraee et $\mathrm{al}^{70}$ that found an increase in corticospinal excitability with increasing stimulation duration up to 24 minutes for tDCS application over the 
motor cortex and decrease or even reversed excitability for stimulation duration at 26, 28, and 30 minutes. ${ }^{70}$ However, the location of stimulation target (ie, frontal cortices versus motor cortices) might contribute to the discrepancy seen in observed effects with prolonged stimulation duration (eg, 20 minutes versus 30 minutes) and thus implied that changes in excitability might vary depending on where in the cortex. Nevertheless, our meta regression results suggested that there was no relationship of observed effect size with duration $(b$-weight $=-0.005, \mathrm{CI}=[-0.019 ; 0.010])$ nor number of sessions ( $b$-weight $=0.005, \mathrm{CI}=[-0.027$; 0.037]). Regarding the number of sessions, the average effect size within the working memory domain for singlesession studies was 0.51 while the average effect size for multi-session studies was 0.49 . These findings demonstrated that the effect size produced from single-session vs multi-session was comparable. Prior research suggests that multi-session studies yield greater effects than single session studies in psychiatric population (eg, addiction and Parkinson's Disease),${ }^{71,72}$ neuropathic pain population, ${ }^{73}$ major depressive disorder, and post-stroke aphasia patients. ${ }^{74}$ However, to the best of our knowledge, there is no study that examines such effects (ie, the optimal number of tDCS stimulation sessions) in a healthy aging sample. As such, due to the small number of single versus multi-session data in our sample and the lack of information in the literature regarding the efficacy of single vs multi-session tDCS in healthy older adults, further investigation regarding the effects of number of sessions for tDCS application in this population is required to help inform specific stimulation recommendation. It is also important to note that this observation was difficult to translate from the working memory domain onto other cognitive domains reported in this review because of the diversity of outcome measures used within the same cognitive domain. Therefore, recommending which current dose parameter will produce the largest effect size for each cognitive domain remains a challenge.

All studies included in this review aimed to stimulate the frontal cortex to improve cognition by placing electrode nearby or over the frontal region following the 10-20 EEG electrode system with various anode and cathode locations. Previous computational modeling studies have investigated the relationship between current distribution and electrode placement and shown the largest current intensity within underlying brain regions is typically found between the electrode pair rather than directly underneath each electrode. ${ }^{75-84}$ Among the studies that reported statistically significant effect sizes, the anode electrodes were placed over the frontal cortex in location F4, F3, or F7 corresponding to the targeted hemisphere for stimulation (eg, anode electrode at F4 to stimulate the right DLPFC region or anode at F3 location to target the left DLPFC). The cathode electrodes were placed either at the head apex $(\mathrm{Cz})$ or in the contralateral area such as supraorbital or extracephalic location (deltoid muscle, cheek). The distance between the anode and cathode electrode has been suggested to have an impact on current distribution inside the head. ${ }^{85}$ However, in the present review, there was no clear conclusion that could be drawn about the influence of the distance between each electrode pair and the resulting effect size in each outcome. In contrast, the included studies may suggest that the direction of current flow indicated by reversing placement of the same electrode pair seems to have a considerable effect on measured outcome. For instance, Harty et al, reported a statistically significant effect size for electrode pair anode-cathode at F4-Cz to target the right DLPFC and improve error awareness via Go/No-go tasks. However, when the anode and cathode location was reversed (eg, Cz-F4) for the same outcome measure, the effects of stimulation in the right DLPFC were not statistically significant. This observation suggests that the directional component of applied electrical field may play a crucial role in determining optimum current dose in tDCS application to elicit observed outcomes which support recent research findings in this topic. ${ }^{86-89}$

\section{tDCS and Cognitive Tasks}

Positive tDCS effects have been found to be state-dependent, and thus, the level of engagement in cognitive tasks may be important to generate desired outcomes. Electrical current from tDCS alone is considered weak and non-specific to enhance synaptic efficacy. Therefore, specific brain regions are targeted by combining tDCS and training to increase sensitivity of the desired brain area, making it more perceptive to applied stimulation..$^{90}$ This mechanism is referred to as functional specificity, where the pairing of activity and selectivity is presumed to enhance neural activity in the targeted brain regions by engaging specific networks to the stimulation, separating them from other ongoing background brain activity. ${ }^{90}$ Online stimulation is performed when the stimulation and cognitive tasks occur at the same time. In this context, applying stimulation during tasks that engage a targeted cognitive domain can potentially produce a synergistic and augmenting stimulation effect. Offline 
stimulation is conducted without pairing of a task, usually before or after task practice. A previous systematic review and meta analyses by Summers et $\mathrm{al}^{29}$ reported that the average effect size in cognitive outcomes was greater in offline stimulation compared to online stimulation. However, only $12 \%$ of the studies included in the previous review employed an offline stimulation. Similar findings were also reported by Hsu et $\mathrm{al}^{39}$ where offline stimulation for tDCS and repetitive transcranial magnetic stimulation (rTMS) were found to be more effective in healthy older adults. However, Hsu et al did not further separate offline versus online effects according to each stimulation modality (ie, tDCS versus rTMS). In contrast, pairing tDCS with cognitive tasks such as an N-Back task to target working memory has been found superior in improving working memory performance compared to applying stimulation prior to task execution. ${ }^{91}$ In the present review, we found that online tDCS application during cognitive tasks did not seem to produce larger effect sizes than offline stimulation. However, this observation should be treated with caution because there were only three studies that performed offline tDCS (average effect size: $1.23 \pm 0.58$ ) included in this review while the remaining studies used online tDCS (average effect size: $0.57 \pm 0.36$ ). Our meta-regression analyses also indicated that the effect of timing (online/offline) was nonsignificant $(b$-weight $=0.0812, \mathrm{CI}=[-0.22 ; 0.38], \mathrm{p}=0.569)$. Further, the lack of information regarding the concurrent activity as participants receiving tDCS in offline cases makes it challenging to directly compare online and offline stimulation outcomes. Overall, across the included thirteen studies, the heterogeneity of reported results makes it difficult to conclude which timing of stimulation delivery with respect to performed tasks (online or offline) would yield the maximum effect size in each cognitive domain.

In addition to stimulation timing with respect to performed tasks, an extended period of cognitive training outside the stimulation period may contribute to improved cognitive outcomes and thus needs to be considered. Prior research suggests that pairing tDCS with cognitive training can prolong the outcome effects compared to performing cognitive training alone. ${ }^{92-95}$ There are four studies in the present review (Nissim et al, 2019, Park et al, 2014, Stephens et al, 2016, Nilsson et al, 2017) that implemented an extended period of cognitive training beyond the stimulation duration aiming to improve working memory performance. The duration of the cognitive training among these studies (40 minutes x 10 sessions, or 40 minutes $\times 20$ sessions, or 30 minutes $\times 10$ sessions) as well as the tDCS parameters used were largely varied with different length of stimulation duration (20 or 30 minutes in each session). Therefore, it is unclear which combination of cognitive training duration and stimulation parameters would produce the greatest effect size for the working memory domain. However, three out of four studies that implemented cognitive training found moderate effect sizes (mean: $0.76+0.31$ ) that were statistically significant $(p<0.05)$, which suggests the promising benefit of pairing cognitive training with tDCS application to improve working memory performance in older adults.

\section{Implications for tDCS Application in Older Adults}

While some studies employed the same tDCS set-up (eg, 1 $\mathrm{mA}, 35 \mathrm{~cm}^{2}$ electrodes placed at $\mathrm{F} 4-\mathrm{Cz}$ ), diverse effect sizes were seen across study outcomes and may largely be influenced by heterogeneity in age-related structural decline across participants. At present, in-vivo measurements to quantify the amount of delivered current in the brain from stimulation are difficult to accomplish. Therefore, computational models have been utilized to estimate current dose in the brain. Recent tDCS modeling studies have shown that interindividual variability in anatomy has an important role in altering delivered tDCS current in the brain. ${ }^{88,96-100}$ In older adults, age-related effects such as brain atrophy and white matter hyperintensities (WMH) were found as important factors that affect modeled field distribution following tDCS. ${ }^{77,101}$ Brain atrophy is commonly described as a shrinkage of cortical structures that occurs with healthy aging. ${ }^{102}$ The rate of atrophy is varied across individuals, and thus, the severity of brain atrophy found across older adult samples can vary. ${ }^{103-106}$ The frontal region, especially the prefrontal cortex, is the first to structurally decline with age. ${ }^{107}$ All included studies in this review aimed to stimulate the frontal cortex. The variation seen in stimulation outcomes may be influenced by individual rates of atrophy in this region. A recent modeling study performed in 587 older adults (mean age: 73.9 years) demonstrates that shrinkage in cortical structures (brain atrophy) can affect current distribution in the brain. ${ }^{77}$ Human brain tissues (white and gray matter) are immersed in cerebrospinal fluid (CSF) which is a better electrical conductor than brain. When brain volume shrinks due to atrophy, the amount of CSF inside the brain cavity is increased causing a higher ratio of CSF compared to brain. Therefore, current 
delivered from the stimulation that reaches the brain cavity would likely travel within CSF causing less current to enter the brain. ${ }^{77,81,108}$ In addition, another recent modeling study investigating WMH in 130 older adults found that the presence of WMH could reduce delivered current in intact brain up to $7 \% .{ }^{101}$ This occurrence suggests that it is difficult to recommend a standardized tDCS parameter that will work for all and, instead, tDCS applications in older adults may need to be tailored to each individual and account for various atrophy and WMH levels across older adult samples. For instance, adjusting current intensity level and using custom electrode placement to target desired brain region in each person. Further, application of one-size-fit-all may need improving for future tDCS application such as through the use of machine learning approaches $^{89}$ to optimize outcomes and reduce interindividual variability observed across tDCS participants.

\section{Study Limitation and Future Direction}

Overall tDCS effects reported in the present review are limited by the heterogeneity of reported tDCS parameters and the overall sample size used across studies. Out of the 13 included studies, those that reported the highest magnitude of an effect also had the smallest sample sizes ( $\mathrm{n}$ range $=10-11$ ). While one of these studies reported a power analysis supporting sufficient power (Manenti et al, 2017), others cited small sample size as a limitation. Under-powered studies, or studies with smaller sample sizes, tend to have inflated effect-sizes. ${ }^{109}$ Therefore, future studies assessing cognitive changes due to tDCS intervention in older adults should have a larger sample size to better estimate the efficacy of tDCS in remediating cognitive decline. While some of the included studies were underpowered, 9 out of 13 studies included in this review had adequately powered sample sizes and still reported medium to large effect sizes, suggesting that even with adequate power, tDCS intervention shows promise as an effective intervention in cognitive aging. In addition, we acknowledge that our snowballing results might be incomplete since we did not use Scopus due to the lack of institutional access. However, Bakkalbasi et al ${ }^{110}$ conducted a study to compare citation tracking tools between Google Scholar, Scopus, and Web of science and concluded that the study could not claim one tool to be the clear winner for all subject matter. An additional limitation that should be considered when interpreting findings from this review is publication bias. Based on our analyses, we found an indication of publication bias within our sample. However, these analyses did not include other papers that may have null findings or findings that do not support their hypothesis that are not published. A recent paper by Murray et $\mathrm{al}^{111}$ assessed 433 randomized controlled trials in JAMA network, and found no evidence to support that direction of findings (ie, supporting or rejecting the null hypothesis) influenced publication. However, null trials may have had to pass different standards to get published and there are no studies commenting on unpublished null papers. ${ }^{112}$ Therefore, it is important to note that the results reported in this meta-analysis needs to be taken with caution and publication bias must remain a consideration when interpreting these conclusions. With regard to study impact, the majority of the included studies have small sample sizes (mean $\mathrm{n}=31 \pm 18$ ), making it difficult to assess the impact on the general population. Studies involving a larger sample size, such as the ongoing Phase III tDCS ACT clinical trial, ${ }^{113}$ are warranted to further assess the potential effect of tDCS to remediate cognitive aging in a healthy older adult population. Further, most studies did not report any metrics of quality assurance to monitor the accuracy and consistency of electrode placement. This is crucial information since any shift in electrode location as little as $1 \mathrm{~cm}$ across stimulation sessions can alter the amount of current entering the brain. ${ }^{84,114-116}$ The exact source of variability is difficult to identify across studies; therefore, future tDCS studies should incorporate characterization of the potential source of variability to explain any unexpected or muddled results. In addition, the present review only included studies that were published up to June 30th, 2020. There may be additional relevant studies that have been published since this date that will need to be included in future systematic reviews of this topic. Further, this review only covers cognitively intact and healthy aging population limited to age 65 and above. This age range might be considered restrictive if brain atrophy occurs in earlier age. The lack of an upper age limit in our search criteria may raise a concern; however, there is no evidence in the literature suggesting that there is a change in the pattern of age-related cognitive decline, but rather a continued decline in cognitive function. ${ }^{117}$ Future meta-analyses can expand the age range to include midlife or focus on cross-sectional and longitudinal studies to further analyze the effects of atrophy on observed cognitive outcomes. Other published meta-analyses performed in healthy young adults have included middle-aged and older adults, without specified age limit. ${ }^{25,38,118,119}$ The lack of meta-analyses conducted exclusively in healthy younger adult cohorts makes it difficult to compare our findings to a young adult population. Therefore, there is an opportunity for future meta-analyses to include exclusively younger populations. Future review studies can also assess the potential benefits of tDCS on cognitive 
performance in populations with mild cognitive impairment (MCI) or Alzheimer's disease (AD).

\section{Conclusion}

In the present systematic review, we reported thirteen independent studies that employed tDCS to improve cognition in a healthy older adult population. We found small to large effect sizes with a weighted average of $0.63(\mathrm{CI}=$ [0.42;0.84]) across all studies reporting effects of active tDCS over sham on various cognitive domains. Reported effect sizes were deemed statistically significant $(\mathrm{p}<0.05)$ in $76.5 \%$ of the experiments within the thirteen studies. While the included studies employed various tDCS parameters and diverse outcome measures were used to assess cognitive performance in different domains, these findings demonstrate an overall positive effect of tDCS delivered to the frontal lobes for remediating cognitive decline in older adults. Thus, these collective data suggest a potential use of tDCS as an effective intervention for cognitive aging that warrants further exploration. Future characterization of inter-individual variability in tDCS dose response is recommended for optimizing tDCS application in older adults and reducing variability in observed outcomes.

\section{Acknowledgments}

This work was supported by the National Institutes of Health/National Institute on Aging/National Heart, Lung, and Blood Institute (R01AG054077, K01AG050707, T32AG020499, T32HL134621, T32AG 61892), the University of Florida McKnight Brain Institute and the McKnight Brain Research Foundation. Research reported in this publication was supported by the University of Florida Clinical and Translational Science Institute, which is supported in part by the NIH National Center for Advancing Translational Sciences under award number UL1TR001427. The content is solely the responsibility of the authors and does not necessarily represent the official views of the National Institutes of Health.

\section{Disclosure}

The authors report no conflicts of interest in this work.

\section{References}

1. Ortman JM, Velkoff VA, Hogan H. An aging nation: the older population in the United States. Econ Stat Adm US Dep Commer. 2014;1964:1-28. doi:10.1016/j.jaging.2004.02.002
2. Sofi F, Valecchi D, Bacci D, et al. Physical activity and risk of cognitive decline: a meta-analysis of prospective studies. $J$ Intern Med. 2011;269(1):107-117. doi:10.1111/j.1365-2796.2010.02281.x

3. Colcombe S, Kramer AF. Fitness effects on the cognitive function of older adults: a meta-analytic study. Psychol Sci. 2003;14 (2):125-130. doi:10.1111/1467-9280.t01-1-01430

4. Bherer L, Peterson MS, Kramer AF, Colcombe S, Erickson K, Becic E. Training effects on dual-task performance: are there age-related differences in plasticity of attentional control? Psychol Aging. 2005;20(4):695-709. doi:10.1037/0882-7974.20. 4.695

5. Erickson KI, Miller DL, Roecklein K. The Aging Hippocampus: interactions between Exercise, Depression, and BDNF. Neurosci. 2012;18(1):82-97. doi:10.1177/1073858410397054

6. Salthouse TA. Selective review of cognitive aging. J Int Neuropsychol Soc. 2010;16(5):754-760. doi:10.1017/S1355 617710000706

7. Schaie KW. Developmental Influences on Adult Intelligence: The Seattle Longitudinal Study. Oxford University Press; 2005.

8. Salthouse TA. Influence of age on practice effects in longitudinal neurocognitive change. Neuropsychology. 2010;24(5):563-572. doi:10.1037/a0019026

9. Harada CN, Natelson Love MC, Triebel KL. Normal cognitive aging. Clin Geriatr Med. 2013;29(4):737-752. doi:10.1016/j. cger.2013.07.002

10. Freitas C, Perez J, Knobel M, et al. Changes in cortical plasticity across the lifespan. Front Aging Neurosci. 2011;3:1-8. doi:10.33 89/fnagi.2011.00005

11. Payne BR, Gross AL, Parisi JM, et al. Modelling longitudinal changes in older adults' memory for spoken discourse: findings from the ACTIVE cohort. Memory. 2014;22(8):990-1001. doi:10.1080/09658211.2013.861916

12. Schupf N, Tang M-X, Albert SM, et al. Decline in cognitive and functional skills increases mortality risk in nondemented elderly. Neurology. 2005;65(8):1218-1226. doi:10.1212/01.wnl.0000180 970.07386.cb

13. Millán-Calenti JC, Tubío J, Pita-Fernández S, Rochette S, Lorenzo T, Maseda A. Cognitive impairment as predictor of functional dependence in an elderly sample. Arch Gerontol Geriatr. 2012;54(1):197-201. doi:10.1016/j.archger.2011.02.010

14. Nitsche MA, Paulus W. Excitability changes induced in the human motor cortex by weak transcranial direct current stimulation. J Physiol. 2000;527(3):633-639. doi:10.1111/j.14 69-7793.2000.t01-1-00633.x

15. Woods AJ, Antal A, Bikson M, et al. A technical guide to tDCS, and related non-invasive brain stimulation tools. Clin Neurophysiol. 2016;127(2):1031-1048. doi:10.1016/j.clinph.20 15.11 .012

16. Gomes-Osman J, Indahlastari A, Fried PJ, et al. Non-invasive Brain Stimulation: probing Intracortical Circuits and Improving Cognition in the Aging Brain. Front Aging Neurosci. 2018;10. doi:10.3389/fnagi.2018.00177

17. Bikson M, Grossman P, Thomas C, et al. Safety of Transcranial Direct Current Stimulation: evidence Based Update 2016. Brain Stimul. 2016;9(5):641-661. doi:10.1016/j.brs.2016.06.004

18. Nitsche MA, Cohen LG, Wassermann EM, et al. Transcranial direct current stimulation: state of the art 2008. Brain Stimul. 2008;1(3):206-223. doi:10.1016/j.brs.2008.06.004

19. Albizu A, Indahlastari A, Woods AJ. Non-invasive Brain Stimulation. In: Encyclopedia of Gerontology and Population Aging. Cham: Springer International Publishing;2019:1-8. doi:10. 1007/978-3-319-69892-2_682-1

20. Kronberg G, Bridi M, Abel T, Bikson M, Parra LC. Direct Current Stimulation Modulates LTP and LTD: activity Dependence and Dendritic Effects. Brain Stimul. 2017;10 (1):51-58. doi:10.1016/j.brs.2016.10.001 
21. Ranieri F, Podda MV, Riccardi E, et al. Modulation of LTP at rat hippocampal CA3-CA1 synapses by direct current stimulation. J Neurophysiol. 2012;107(7):1868-1880. doi:10.1152/jn.00319.2011

22. Kronberg G, Rahman A, Sharma M, Bikson M, Parra LC. Direct current stimulation boosts hebbian plasticity in vitro. Brain Stimul. 2020;13(2):287-301. doi:10.1016/j.brs.2019.10.014

23. Podda MV, Cocco S, Mastrodonato A, et al. Anodal transcranial direct current stimulation boosts synaptic plasticity and memory in mice via epigenetic regulation of Bdnf expression. Sci Rep 2016;6(1). doi:10.1038/srep22180

24. Marquez-Ruiz J, Leal-Campanario R, Sanchez-Campusano R, et al. Transcranial direct-current stimulation modulates synaptic mechanisms involved in associative learning in behaving rabbits. Proc Natl Acad Sci U S A. 2012;109(17):6710-6715. doi:10. 1073/pnas.1121147109

25. Imburgio MJ, Orr JM. Effects of prefrontal tDCS on executive function: methodological considerations revealed by meta-analysis. Neuropsychologia. 2018;117:156-166. doi:10.10 16/j.neuropsychologia.2018.04.022

26. Fregni F, Liguori P, Fecteau S, Nitsche MA, Pascual-Leone A, Boggio PS. Cortical stimulation of the prefrontal cortex with transcranial direct current stimulation reduces cue-provoked smoking craving: a randomized, sham-controlled study. J Clin Psychiatry. 2008;69(1):32-40. doi:10.4088/JCP.v69n0105

27. Berryhill ME, Peterson DJ, Jones KT, Stephens JA. Hits and misses: leveraging tDCS to advance cognitive research. Front Psychol. 2014;5:800. doi:10.3389/fpsyg.2014.00800

28. Tatti E, Rossi S, Innocenti I, Rossi A, Santarnecchi E. Noninvasive brain stimulation of the aging brain: state of the art and future perspectives. Ageing Res Rev. 2016. doi:10.1016/j.arr. 2016.05.006

29. Summers JJ, Kang N, Cauraugh JH. Does transcranial direct current stimulation enhance cognitive and motor functions in the ageing brain? A systematic review and meta- analysis. Ageing Res Rev. 2016;25:42-54. doi:10.1016/j.arr.2015.11.004

30. Keeser D, Meindl T, Bor J, et al. Prefrontal Transcranial Direct Current Stimulation Changes Connectivity of Resting-State Networks during fMRI. J Neurosci. 2011;31(43):15284-15293. doi:10.1523/JNEUROSCI.0542-11.2011

31. Nissim NR, O'Shea A, Indahlastari A, et al. Effects of in-Scanner Bilateral Frontal tDCS on Functional Connectivity of the Working Memory Network in Older Adults. Front Aging Neurosci. 2019;11. doi:10.3389/fnagi.2019.00051

32. Nissim NR, O'Shea A, Indahlastari A, et al. Effects of transcranial direct current stimulation paired with cognitive training on functional connectivity of the working memory network in older adults. Front Aging Neurosci. 2019;11. doi:10.3389/fnagi.2019. 00340

33. Manenti R, Sandrini M, Gobbi E, Binetti G, Cotelli M. Effects of transcranial direct current stimulation on episodic memory in amnestic mild cognitive impairment: a pilot study. J Gerontol B Psychol Sci Soc Sci. 2020;75(7):1403-1413. doi:10.1093/geronb/gby 134

34. Perceval G, Flöel A, Meinzer M. Can transcranial direct current stimulation counteract age-associated functional impairment? Neurosci Biobehav Rev. 2016;65:157-172. doi:10.1016/j. neubiorev.2016.03.028

35. Aubertin-Leheudre M, Woods AJ, Anton S, Cohen R, Pahor M. Frailty Clinical Phenotype: A Physical and Cognitive Point of View. Vol. 83. 2015. doi:10.1159/000382061

36. Diehl M, Marsiske M, Horgas AL, Rosenberg A, Saczynski JS, Willis SL. The revised observed tasks of daily living: a performance-based assessment of everyday problem solving in older adults. J Appl Gerontol. 2005. doi:10.1177/073346480427 3772
37. Thomas KR, Marsiske M. Verbal prompting to improve everyday cognition in MCI and unimpaired older adults. Neuropsychology. 2014;28(1):123-134. doi:10.1037/neu0000039

38. Dedoncker J, Brunoni AR, Baeken C, Vanderhasselt MA. A systematic review and meta-analysis of the effects of transcranial direct current stimulation (tDCS) over the dorsolateral prefrontal cortex in healthy and neuropsychiatric samples: influence of stimulation parameters. Brain Stimul. 2016;9(4):501-517. doi:10.1016/j.brs.2016.04.006

39. Hsu W-Y, Ku Y, Zanto TP, Gazzaley A. Effects of noninvasive brain stimulation on cognitive function in healthy aging and Alzheimer's disease: a systematic review and meta-analysis. Neurobiol Aging. 2015;36(8):2348-2359. doi:10.1016/j.neurobiolaging.2015.04.016

40. Tremblay S, Lepage JF, Latulipe-Loiselle A, Fregni F, PascualLeone A, Théoret H. The uncertain outcome of prefrontal tDCS. Brain Stimul. 2014;7(6):773-783. doi:10.1016/j.brs.2014.10.003

41. Horvath JC, Forte JD, Carter O. Quantitative Review Finds No Evidence of Cognitive Effects in Healthy Populations from Single-Session Transcranial Direct Current Stimulation (tDCS). Brain Stimul. 2015;8(3):535-550. doi:10.1016/j.brs.2015.01.400

42. Inagawa $T$, Narita $Z$, Sugawara $N$, et al. A meta-analysis of the effect of multisession transcranial direct current stimulation on cognition in dementia and mild cognitive impairment. Clin EEG Neurosci. 2019;50(4):273-282. doi:10.1177/1550059418800889

43. Rajji TK. Transcranial Magnetic and. Electrical stimulation in alzheimer's disease and mild cognitive impairment: a review of randomized controlled trials. Clin Pharmacol Ther. 2019. doi:10.1002/cpt.1574

44. McAvinue LP, Habekost T, Johnson KA, et al. Sustained attention, attentional selectivity, and attentional capacity across the lifespan. Attention Perception Psychophys. 2012;74(8):157 0-1582. doi:10.3758/s13414-012-0352-6

45. Glisky EL. Changes in Cognitive Function in Human Aging. In: Brain Aging. CRC Press; 2007:3-20. doi:10.1201/97814200 05523-1

46. Verhaeghen P, Salthouse TA. Meta-analyses of age-cognition relations in adulthood: estimates of linear and nonlinear age effects and structural models. Psychol Bull. 1997;122(3):2 31-249. doi:10.1037/0033-2909.122.3.231

47. Salthouse TA. When does age-related cognitive decline begin? Neurobiol Aging. 2009;30(4):507-514. doi:10.1016/j. neurobiolaging.2008.09.023

48. Finkel D, Reynolds CA, McArdle JJ, Pedersen NL. Age changes in processing speed as a leading indicator of cognitive aging. Psychol Aging. 2007;22(3):558-568. doi:10.1037/0882-7974.22. 3.558

49. Arent SM, Landers DM, Etnier JL. The effects of exercise on mood in older adults: a meta-analytic review. J Aging Phys Act. 2000;8(4):407-430. doi:10.1123/japa.8.4.407

50. Lee SB, Oh JH, Park JH, Choi SP, Wee JH. Differences in youngest-old, middle-old, and oldest-old patients who visit the emergency department. Clin Exp Emerg Med. 2018;5(4):249-255. doi:10.15441/ceem.17.261

51. Centers of Disease Control and Prevention. Subjective Cognitive Decline - A Public Health Issue. US Dep Heal Hum Serv; 2018.

52. Nations U, of Economic D, Affairs S, Division P. World Population Ageing 2019: Highlights; 2019.

53. Higgins JPT, Altman DG, Gøtzsche PC, et al. The Cochrane Collaboration's tool for assessing risk of bias in randomised trials. BMJ. 2011;343(oct18 2):d5928-d5928. doi:10.1136/bmj.d5928

54. Sterne JAC, Savović J, Page MJ, et al. RoB 2: a revised tool for assessing risk of bias in randomised trials. BMJ. 2019;366:14898. doi:10.1136/bmj.14898

55. Egger M, Smith GD, Schneider M, Minder C. Bias in meta-analysis detected by a simple, graphical test. $\mathrm{Br} \mathrm{Med} J$. 1997;315(7109):629-634. doi:10.1136/bmj.315.7109.629 
56. Duval S, Tweedie R. A Nonparametric "Trim and Fill" Method of Accounting for Publication Bias in Meta-Analysis. $J$ Am Stat Assoc. 2000. doi:10.1080/01621459.2000.10473905

57. Duval S, Tweedie R. Trim and fill: a simple funnel-plot-based method of testing and adjusting for publication bias in meta-analysis. Biometrics. 2000;56(2):455-463. doi:10.1111/ j.0006-341X.2000.00455.x

58. Brosnan MB, Arvaneh M, Harty S, et al. Prefrontal modulation of visual processing and sustained attention in aging, a tDCS-EEG coregistration approach. $J$ Cogn Neurosci. 2018;30(11):16 30-1645. doi:10.1162/jocn_a_01307

59. Manenti R, Sandrini M, Gobbi E, et al. Strengthening of existing episodic memories through non-invasive stimulation of prefrontal cortex in older adults with subjective memory complaints. Front Aging Neurosci. 2017;9. doi:10.3389/fnagi.2017.00401

60. Medvedeva A, Materassi M, Neacsu V, et al. Effects of Anodal Transcranial Direct Current Stimulation Over the Ventrolateral Prefrontal Cortex on Episodic Memory Formation and Retrieval. Cereb Cortex. 2019;29(2):657-665. doi:10.1093/cercor/bhx347

61. Sandrini M, Manenti R, Brambilla M, Cobelli C, Cohen LG, Cotelli M. Older adults get episodic memory boosting from noninvasive stimulation of prefrontal cortex during learning. Neurobiol Aging. 2016;39:210-216. doi:10.1016/j.neurobiolaging.2015.12.010

62. Brosnan MB, Demaria G, Petersen A, Dockree PM, Robertson IH, Wiegand I. Plasticity of the Right-Lateralized Cognitive Reserve Network in Ageing. Cereb Cortex. 2018;28 (5):1749-1759. doi:10.1093/cercor/bhx085

63. Tan A Effects of primed anodal transcranial direct current stimulation on the psychomotor function of older adults. Dr Diss Murdoch Univ. 2016.

64. Cespón J, Rodella C, Rossini PM, Miniussi C, Pellicciari MC. Anodal transcranial direct current stimulation promotes frontal compensatory mechanisms in healthy elderly subjects. Front Aging Neurosci. 2017;9:420. doi:10.3389/fnagi.2017.00420

65. Nilsson J, Lebedev AV, Lövdén M. No Significant Effect of Prefrontal tDCS on Working Memory Performance in Older Adults. Front Aging Neurosci. 2015;7. doi:10.3389/fnagi.2015.00230

66. Nilsson J, Lebedev AV, Rydström A, Lövdén M. Direct-Current Stimulation Does Little to Improve the Outcome of Working Memory Training in Older Adults. Psychol Sci. 2017;28 (7):907-920. doi:10.1177/0956797617698139

67. Park S-H, Seo J-H, Kim Y-H, Ko M-H. Long-term effects of transcranial direct current stimulation combined with computer-assisted cognitive training in healthy older adults. Neuroreport. 2014;25 (2):122-126. doi:10.1097/WNR.0000000000000080

68. Stephens JA, Berryhill ME. Older adults improve on everyday tasks after working memory training and neurostimulation. Brain Stimul. 2016;9(4):553-559. doi:10.1016/j.brs.2016.04.001

69. Harty S, Robertson IH, Miniussi C, et al. Transcranial direct current stimulation over right dorsolateral prefrontal cortex enhances error awareness in older age. $J$ Neurosci. 2014;34 (10):3646-3652. doi:10.1523/JNEUROSCI.5308-13.2014

70. Hassanzahraee M, Nitsche MA, Zoghi M, Jaberzadeh S. Determination of anodal tDCS duration threshold for reversal of corticospinal excitability: an investigation for induction of counter-regulatory mechanisms. Brain Stimul. 2020;13(3):8 32-839. doi:10.1016/j.brs.2020.02.027

71. Song S, Zilverstand A, Gui W, Li H, Zhou X. Effects of single-session versus multi-session non-invasive brain stimulation on craving and consumption in individuals with drug addiction, eating disorders or obesity: a meta-analysis. Brain Stimul. 2019;12(3):606-618. doi:10.1016/j.brs.2018.12.975

72. Orrù $\mathrm{G}$, Baroni $\mathrm{M}$, Cesari V, Conversano $\mathrm{C}$, Hitchcott $\mathrm{PK}$, Gemignani A. The effect of single and repeated tDCS sessions on motor symptoms in Parkinson's disease: a systematic review. Arch Ital Biol. 2019;(2):89-101. doi:10.12871/00039829201925
73. David MCMM, Moraes AAD, Costa MLD, Franco CIF. Transcranial direct current stimulation in the modulation of neuropathic pain: a systematic review. Neurol Res. 2018;40 (7):557-565. doi:10.1080/01616412.2018.1453190

74. Fregni F, El-Hagrassy MM, Pacheco-Barrios K, et al. Evidencebased guidelines and secondary meta-analysis for the use of transcranial direct current stimulation (tDCS) in neurological and psychiatric disorders. Int J Neuropsychopharmacol. 2020. doi:10.1093/ijnp/pyaa051

75. Bikson M, Rahman A, Datta A. Computational Models of Transcranial Direct Current Stimulation. Clin EEG Neurosci. 2012;43(3):176-183. doi:10.1177/1550059412445138

76. Wagner T, Fregni F, Fecteau S, Grodzinsky A, Zahn M, PascualLeone A. Transcranial direct current stimulation: a computer-based human model study. Neuroimage. 2007;35(3):1113-1124. doi:10. 1016/j.neuroimage.2007.01.027

77. Indahlastari A, Albizu A, O'Shea A, et al. Modeling transcranial electrical stimulation in the aging brain. Brain Stimul. 2020;13 (3):664-674. doi:10.1016/j.brs.2020.02.007

78. Huang Y, Dmochowski JP, Su Y, Datta A, Rorden C, Parra LC. Automated MRI segmentation for individualized modeling of current flow in the human head. J Neural Eng. 2013;10 (6):066004. doi:10.1088/1741-2560/10/6/066004

79. Windhoff M, Opitz A, Thielscher A. Electric field calculations in brain stimulation based on finite elements: an optimized processing pipeline for the generation and usage of accurate individual head models. Hum Brain Mapp. 2013;34(4):923-935. doi:10. $1002 / \mathrm{hbm} .21479$

80. Ruffini G, Wendling F, Merlet I, et al. Transcranial current brain stimulation (tCS): models and technologies. IEEE Trans Neural Syst Rehabil Eng. 2013;21(3):333-345. doi:10.1109/TNSRE.20 12.2200046

81. Mahdavi S, Towhidkhah F. Computational human head models of tDCS: influence of brain atrophy on current density distribution. Brain Stimul. 2018;11(1):104-107. doi:10.1016/j. brs.2017.09.013

82. Thomas C, Datta A, Woods A Effect of aging on cortical current flow due to transcranial direct current stimulation: considerations for safety. In: Proceedings of the Annual International Conference of the IEEE Engineering in Medicine and Biology Society, EMBS.; 2018. doi:10.1109/EMBC.2018.8513014

83. Sadleir RJ, Vannorsdall TD, Schretlen DJ, Gordon B. Target optimization in transcranial direct current stimulation. Front Psychiatry. 2012;3. doi:10.3389/fpsyt.2012.00090

84. Opitz A, Yeagle E, Thielscher A, Schroeder C, Mehta AD, Milham MP. On the importance of precise electrode placement for targeted transcranial electric stimulation. Neuroimage. 2018;181:560-567. doi:10.1016/j.neuroimage.2018.07.027

85. Moliadze V, Antal A, Paulus W. Electrode-distance dependent after-effects of transcranial direct and random noise stimulation with extracephalic reference electrodes. Clin Neurophysiol. 2010;121(12):2165-2171. doi:10.1016/j.clinph.2010.04.033

86. Rawji V, Ciocca M, Zacharia A, et al. tDCS changes in motor excitability are specific to orientation of current flow. Brain Stimul. 2018;11(2):289-298. doi:10.1016/j.brs.2017.11.001

87. Jacobson L, Koslowsky M, Lavidor M. TDCS polarity effects in motor and cognitive domains: a meta-analytical review. Exp Brain Res. 2012;216(1):1-10. doi:10.1007/s00221-011-2891-9

88. Antonenko D, Thielscher A, Saturnino GB, et al. Towards precise brain stimulation: is electric field simulation related to neuromodulation? Brain Stimul. 2019;12(5):1159-1168. doi:10. 1016/j.brs.2019.03.072

89. Albizu A, Fang R, Indahlastari A, et al. Machine learning and individual variability in electric field characteristics predict tDCS treatment response. Brain Stimul. 2020;13(6):1753-1764. doi:10. 1016/j.brs.2020.10.001 
90. Bikson M, Rahman A. Origins of specificity during tDCS: anatomical, activity-selective, and input-bias mechanisms. Front Hum Neurosci. 2013;7. doi:10.3389/fnhum.2013.00688

91. Martin DM, Liu R, Alonzo A, Green M, Loo CK. Use of transcranial direct current stimulation (tDCS) to enhance cognitive training: effect of timing of stimulation. Exp Brain Res. 2014;232 (10):3345-3351. doi:10.1007/s00221-014-4022-x

92. Katz B, Au J, Buschkuehl M, et al. Individual differences and long-term consequences of tDCS-augmented cognitive training. J Cogn Neurosci. 2017;29(9):1498-1508. doi:10.1162/jocn_a_01115

93. Ruf SP, Fallgatter AJ, Plewnia C. Augmentation of working memory training by transcranial direct current stimulation (tDCS). Sci Rep. 2017;7(1):876. doi:10.1038/s41598-017-01055-1

94. Martin DM, Liu R, Alonzo A, et al. Can transcranial direct current stimulation enhance outcomes from cognitive training? A randomized controlled trial in healthy participants. Int J Neuropsychopharmacol. 2013;16(9):1927-1936. doi:10.1017/S1461145713000539

95. Andrews SC, Hoy KE, Enticott PG, Daskalakis ZJ, Fitzgerald PB. Improving working memory: the effect of combining cognitive activity and anodal transcranial direct current stimulation to the left dorsolateral prefrontal cortex. Brain Stimul. 2011;4(2):84-89. doi:10.1016/j.brs.2010.06.004

96. Indahlastari A, Chauhan M, Sadleir RJ. Benchmarking transcranial electrical stimulation finite element models: a comparison study. J Neural Eng. 2019;16(2):026019. doi:10.1088/1741-2552/aafbbd

97. Datta A, Truong D, Minhas P, Parra LC, Bikson M. Interindividual variation during transcranial direct current stimulation and normalization of dose using MRI-derived computational models. Front Psychiatry. 2012;3. doi:10.3389/fpsyt.2012.00091

98. Caulfield KA, Indahlastari A, Nissim NR, et al. Electric field strength from prefrontal transcranial direct current stimulation determines degree of working memory response: a potential application of reverse-calculation modeling? Neuromodulation Technol Neural Interface. 2020. doi:10.1111/ner.13342

99. Huang Y, Datta A, Bikson M, Parra LC. Realistic vOlumetricApproach to Simulate Transcranial Electric Stimulation ROAST - a fully automated open-source pipeline. J Neural Eng. 2019;16(5):056006. doi:10.1088/1741-2552/ab208d

100. Opitz A, Paulus W, Will S, Antunes A, Thielscher A. Determinants of the electric field during transcranial direct current stimulation. Neuroimage. 2015;109:140-150. doi:10.1016/j. neuroimage.2015.01.033

101. Indahlastari A, Albizu A, Boutzoukas EM, O’Shea A, Woods AJ. White matter hyperintensities affect transcranial electrical stimulation in the aging brain. Brain Stimul. 2021;14(1):69-73. doi:10.1016/j.brs.2020.11.009

102. Indahlastari A, Woods AJ. Brain Atrophy. In: Gu D, Dupre ME editors. Encyclopedia of Gerontology and Population Aging. Cham: Springer International Publishing; 2019:1-3. doi:10.1007/ 978-3-319-69892-2_667-1

103. Double KL, Halliday GM, Krill JJ, et al. Topography of brain atrophy during normal aging and alzheimer's disease. Neurobiol Aging. 1996;17(4):513-521. doi:10.1016/0197-4580(96)00005-X

104. Fjell AM, Walhovd KB, Fennema-Notestine C, et al. One-Year Brain Atrophy Evident in Healthy Aging. J Neurosci. 2009;29 (48):15223-15231. doi:10.1523/JNEUROSCI.3252-09.2009

105. Lemaitre H, Goldman AL, Sambataro F, et al. Normal age-related brain morphometric changes: nonuniformity across cortical thickness, surface area and gray matter volume? Neurobiol Aging. 2012;33 (3):617.e1-617.e9. doi:10.1016/j.neurobiolaging.2010.07.013
106. Ge Y, Grossman RI, Babb JS, Rabin ML, Mannon LJ, Kolson DL. Age-related total gray matter and white matter changes in normal adult brain. Part I: volumetric MR imaging analysis. AJNR Am J Neuroradiol. 2002;23(8):1327-1333.

107. Nissim NR, O'Shea AM, Bryant V, Porges EC, Cohen R, Woods AJ. Frontal structural neural correlates of working memory performance in older adults. Front Aging Neurosci. 2017;08. doi:10.3389/fnagi.2016.00328

108. Thomas C, Datta A, Woods A. Effect of aging on current flow due to transcranial direct current stimulation. Brain Stimul. 2017;10 (2):469. doi:10.1016/j.brs.2017.01.374

109. Kühberger A, Fritz A, Scherndl T. Publication bias in psychology: a diagnosis based on the correlation between effect size and sample size. PLoS One. 2014;9(9):e105825. doi:10.1371/journal. pone. 0105825

110. Bakkalbasi N, Bauer K, Glover J, Wang L. Three options for citation tracking: google Scholar, Scopus and Web of Science. Biomed Digit Libr. 2006;3(1). doi:10.1186/1742-5581-3-7

111. Murray SB, Heathers JA, Schauer RM, et al. Postpublication Metrics of Randomized Clinical Trials with and Without Null Findings. JAMA. 2019;321(18):1825. doi:10.1001/jama.2019.2994

112. Cristea IA, Gentili C. Publication bias in trials with and without null findings. JAMA. 2019;322(12):1213. doi:10.1001/jama.2019.11140

113. Woods AJ, Cohen R, Marsiske M, Alexander GE, Czaja SJ, Wu S. Augmenting cognitive training in older adults (The ACT Study): design and Methods of a Phase III tDCS and cognitive training trial. Contemp Clin Trials. 2018;65:19-32. doi:10.1016/j.cct.2017.11.017

114. Indahlastari A, Albizu A, Nissim NR, Traeger KR, O'Shea A, Woods AJ. Methods to monitor accurate and consistent electrode placements in conventional transcranial electrical stimulation. Brain Stimul. 2019;12(2):267-274. doi:10.1016/j.brs.2018.10.016

115. Woods AJ, Bryant V, Sacchetti D, Gervits F, Hamilton R. Effects of electrode drift in transcranial direct current stimulation. Brain Stimul. 2015;8(3):515-519. doi:10.1016/j.brs.2014.12.007

116. De Witte S, Klooster D, Dedoncker J, Duprat R, Remue J, Baeken C. Left prefrontal neuronavigated electrode localization in tDCS: 10-20 EEG system versus MRI-guided neuronavigation. Psychiatry Res Neuroimaging. 2018;274:1-6. doi:10.1016/j. pscychresns.2018.02.001

117. Brayne C, Spiegelhalter DJ, Dufouil C, et al. Estimating the true extent of cognitive decline in the old old. J Am Geriatr Soc. 1999;47 (11):1283-1288. doi:10.1111/j.1532-5415.1999.tb07426.x

118. Hill AT, Fitzgerald PB, Hoy KE. Effects of anodal transcranial direct current stimulation on working memory: a systematic review and meta-analysis of findings from healthy and neuropsychiatric populations. Brain Stimul. 2016;9(2):197-208. doi:10.1016/j.brs.2015.10.006

119. Galli G, Vadillo MA, Sirota M, Feurra M, Medvedeva A. A systematic review and meta-analysis of the effects of transcranial direct current stimulation (tDCS) on episodic memory. Brain Stimul. 2019;12(2):231-241. doi:10.1016/j.brs.2018.11.008

120. Moher D, Liberati A, Tetzlaff J, Altman DG. Preferred reporting items for systematic reviews and meta-analyses: The PRISMA statement. PLoS Medicine. 2009;6(7):e1000097. doi:10.1371/ journal.pmed.1000097 


\section{Publish your work in this journal}

Neuropsychiatric Disease and Treatment is an international, peerreviewed journal of clinical therapeutics and pharmacology focusing on concise rapid reporting of clinical or pre-clinical studies on a range of neuropsychiatric and neurological disorders. This journal is indexed on PubMed Central, the 'PsycINFO' database and CAS, and is the official journal of The International Neuropsychiatric Association (INA). The manuscript management system is completely online and includes a very quick and fair peer-review system, which is all easy to use. Visit http://www.dovepress.com/testimonials.php to read real quotes from published authors.

Submit your manuscript here: https://www.dovepress.com/neuropsychiatric-disease-and-treatment-journal 